\title{
AUTOMORPHIC IMAGES OF COMMUTATIVE SUBSPACE LATTICES
}

\author{
K. J. HARRISON AND W. E. LONGSTAFF
}

\begin{abstract}
Let $C(H)$ denote the lattice of all (closed) subspaces of a complex, separable Hilbert space $H$. Let $(\mathrm{AC})$ be the following condition that a subspace lattice $₹ \subseteq C(H)$ may or may not satisfy:

$\mathcal{F}=\phi(\mathcal{L})$ for some lattice automorphism $\phi$ of $C(H)$ and some commutative subspace lattice $\mathcal{L} \subseteq C(H)$.

Then $\mathcal{F}$ satisfies $(\mathrm{AC})$ if and only if $\mathcal{F} \subseteq B$ for some Boolean algebra subspace lattice $B \subseteq C(H)$ with the property that, for every $K, L \in B$, the vector sum $K+L$ is closed. If $₹$ is finite, then $₹$ satisfies (AC) if and only if $₹$ is distributive and $K+L$ is closed for every $K, L \in \mathcal{F}$. In finite dimensions $\mathcal{F}$ satisfies (AC) if and only if $\mathcal{F}$ is distributive. Every $\mathcal{F}$ satisfying (AC) is reflexive. For such $\mathcal{F}$, given vectors $x, y \in H$, the solvability of the equation $T x=y$ for $T \in \operatorname{Alg} \mathcal{F}$ is investigated.
\end{abstract}

1. Preliminaries. Throughout this paper $H$ will denote a complex, separable, nonzero Hilbert space. The inner product on $H$ is denoted $(\cdot \mid \cdot)$. Let $B(H)$ denote the set of (bounded, linear) operators acting on $H$ and, for $T \in B(H)$, let $R(T)$ denote the range of $T$. Let $C(H)$ denote the set of (closed) subspaces of $H$. By a lattice of subspaces of $H$ is meant a subset $₹ \subseteq C(H)$ which is closed under intersections and closed linear spans, i.e., $M, N \in \mathcal{F}$ implies $M \cap N \in \mathcal{F}$ and $M \vee N \in$ F. A lattice of subspaces $\mathcal{F}$ is complete if $\bigcap M_{\alpha} \in \mathcal{F}$ and $\bigvee M_{\alpha} \in \mathcal{F}$ for every family $\left\{M_{\alpha}\right\}$ of elements of $\mathcal{F}$; it is commutative if $P_{K} P_{L}=P_{L} P_{K}$ for every $K, L \in \mathcal{F}\left(P_{K}\right.$ denotes the orthogonal projection with range $K)$. A subset $\mp \subseteq C(H)$ is strongly closed if $\left\{P_{K}: K \in \mathcal{F}\right\}$ is closed in the strong operator topology. By a subspace lattice on $H$ is meant a complete lattice of subspaces containing (0) and $H$. As

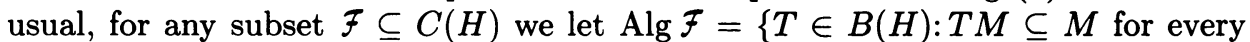
$M \in \mathcal{F}\}$ and let Lat $A=\{N \in C(H): T N \subseteq N$ for every $T \in A\}$ for any subset

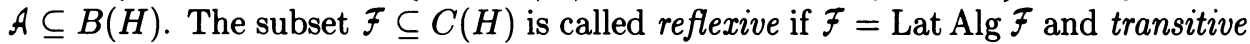
if Lat Alg $\bar{F}=C(H)$. An automorphism $\phi$ of $C(H)$ is a bijection of $C(H)$ onto itself satisfying $M \subseteq N$ if and only if $\phi(M) \subseteq \phi(N)$. Every automorphism $\phi$ satisfies $\phi\left(\bigcap M_{\alpha}\right)=\bigcap \phi\left(M_{\alpha}\right)$ and $\phi\left(\bigvee M_{\alpha}\right)=\bigvee \phi\left(M_{\alpha}\right)$ for every family $\left\{M_{\alpha}\right\} \subseteq C(H)$.

The image $\phi(\mathcal{L})$ of a subspace lattice $\mathcal{L}$ under an automorphism $\phi$ of $C(H)$ is obviously a subspace lattice. If $S \in B(H)$ is invertible, $M \rightarrow S M(M \in C(H))$ defines an automorphism. The image of any subset $₹ \subseteq C(H)$ under this automorphism will be denoted $S ₹$. Much is known about commutative subspace lattices (for example, see $[\mathbf{1}, \mathbf{6}]$ ). It is easy to show that commutativity is not preserved by automorphisms. (For example, let $H=K \oplus K, \mathcal{L}=\{(0), K \oplus(0),(0) \oplus K, H\}$, and let $\phi$ be the automorphism induced by the invertible operator $S \in B(H)$ defined

Received by the editors June 12, 1985.

1980 Mathematics Subject Classification. Primary 46C10; Secondary 06A35.

(C) 1986 American Mathematical Society $0002-9947 / 86 \$ 1.00+\$ .25$ per page 
by $S(x, y)=(x+y, y)$, so that $\phi: M \rightarrow S M(M \subseteq C(H))$.) Denote by (AC) the following condition that a subspace lattice $₹ \subseteq C(H)$ may or may not satisfy:

$$
\begin{aligned}
& \mathcal{F}=\phi(\mathcal{L}) \text { for some lattice automorphism } \phi \text { of } C(H) \\
& \text { and some commutative subspace lattice } \mathcal{L} \text { on } H \text {. }
\end{aligned}
$$

Every commutative subspace lattice on $H$ is reflexive. It is shown in [9] that, if $H$ is infinite-dimensional, automorphisms of $C(H)$ preserve reflexivity (and transitivity). This result is extended to finite-dimensional spaces below. Thus every subspace lattice satisfying condition (AC) is reflexive. A subspace lattice $\mathcal{F}$ is called distributive if $K \cap(L \vee M)=(K \cap L) \vee(K \cap M)$ holds for every $K, L, M \in \mathcal{F}$. It is well known that commutative subspace lattices are distributive. Clearly, if $\mp$ satisfies $(\mathrm{AC})$, then $\mathcal{}$ is distributive. We show that the converse is true in fiinte dimensions. A subspace lattice $B$ is complemented if, for every $K \in B$, there exists an element $K^{\prime} \in B$ such that $K \cap K^{\prime}=(0)$ and $K \vee K^{\prime}=H$. Call $B$ a Boolean algebra if it is complemented and distributive. In Boolean algebras, the complement $K^{\prime}$ of any element $K$ is unique. It is shown that a subspace lattice

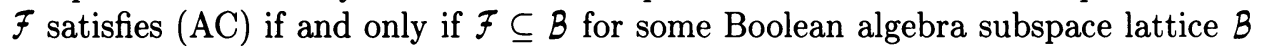
with the property that, for every $K, L \in B$, the vector sum $K+L$ is closed. Also, a finite subspace lattice $\mathcal{F}$ satisfies (AC) if and only if $\mathcal{F}$ is distributive and $K+L$ is closed for every $K, L \in \mathcal{F}$. We conclude with an investigation of the solvability of the equation $T x=y$ for $T \in A \lg \mathcal{F}$, where $x, y$ are given vectors and $\mathcal{F}$ is a given subspace lattice satisfying (AC). Most of the results, notation and terminology we use concerning abstract lattices can be found in [7].

2. Main results. We begin by briefly describing a Boolean notion, applicable to subspaces, that has been studied by several authors in connection with the theory of spectral operators (see [8]). A Boolean algebra of projections in $H$ [8, p. 1928] is a family $\mathcal{Q}$ of commuting bounded idempotents on $H$, containing 0 and 1 , with the property that $\mathcal{Q}$ contains $E F, E+F-E F$ and $1-E$ whenever it contains $E$ and $F$. A Boolean algebra $\mathcal{Q}$ of projections [8, p. 2195] is bounded if there exists $c>0$ such that $\|E\| \leq c$ for every $E \in \mathcal{Q}$, and $\mathcal{Q}$ is complete if, for every family $\left\{E_{\alpha}\right\} \subseteq \mathcal{Q}$, there exist elements $E, F \in \mathcal{Q}$ such that $\bigvee R\left(E_{\alpha}\right)=R(E)$ and $\cap R\left(E_{\alpha}\right)=R(F)$.

PROPOSITION 1. If $\mathcal{Q}$ is a complete Boolean algebra of projections in $H$, then $B=\{R(E): E \in \mathcal{Q}\}$ is a Boolean algebra subspace lattice on $H$ in which all vector sums are closed, i.e., $K+L$ is closed for every $K, L \in B$. Conversely, if $B$ is a Boolean algebra subspace lattice on $H$ in which all vector sums are closed, then $\mathcal{Q}=\left\{Q_{K}: K \in B\right\}$, where $Q_{K}$ denotes the (not necessarily orthogonal) projection onto $K$ along its complement $K^{\prime}$, is a complete Boolean algebra of projections in $H$.

Proof. First, let $B=\{R(E): E \in \mathcal{Q}\}$ with $\mathcal{Q}$ a complete Boolean algebra of projections. Clearly $B$ is a subspace lattice. Since $R(E)+R(F)=R(E+F-E F)$ for every $E, F \in \mathcal{Q}$, all vector sums in $B$ are closed. Since also $R(E) \cap R(F)=R(E F)$, $B$ is complemented and distributive.

Conversely, let $B$ be a Boolean algebra subspace lattice on $H$ in which all vector sums are closed. Let $\mathcal{Q}=\left\{Q_{K}: K \in B\right\}$, where $Q_{K}$ denotes the projection onto $K$ along $K^{\prime}$. Let $K, L \in B$ and let $x \in H$. Then $\left(1-Q_{K \cap L}\right) x \in(K \cap L)^{\prime}=K^{\prime}+L^{\prime}$, so $Q_{K}\left(1-Q_{K \cap L}\right) x=Q_{K} y$ for some $y \in L^{\prime}$. But $Q_{K}\left(1-Q_{K \cap L}\right) x=y-\left(1-Q_{K}\right) y$ 
shows that

$$
Q_{K}\left(1-Q_{K \cap L}\right) x \in K \cap\left(L^{\prime}+K^{\prime}\right)=\left(K \cap L^{\prime}\right)+\left(K \cap K^{\prime}\right)=K \cap L^{\prime} .
$$

Thus $Q_{L} Q_{K}\left(1-Q_{K \cap L}\right) x=0$. Hence $Q_{L} Q_{K}\left(1-Q_{K \cap L}\right)=Q_{L} Q_{K}-Q_{K \cap L}=0$, so $\mathcal{Q}$ is commutative. Clearly $0,1 \in \mathcal{Q}$. If $K, L \in \mathcal{B}$, then $Q_{K}+Q_{L}-Q_{K} Q_{L}=Q_{K+L}$ since $Q_{K}+Q_{L}-Q_{K} Q_{L}$ and $Q_{K+L}$ are commuting idempotents with the same ranges. Since also $1-Q_{K}=Q_{K^{\prime}}$ for every $K \in B$, it now follows that $\mathcal{Q}$ is a Boolean algebra of projections. For every family $\left\{K_{\alpha}\right\} \subseteq B, \bigvee R\left(Q_{K_{\alpha}}\right)=\bigvee K_{\alpha}=R\left(Q_{\vee K_{\alpha}}\right)$ and $\cap R\left(Q_{K_{\alpha}}\right)=\bigcap K_{\alpha}=R\left(Q_{\cap K_{\alpha}}\right)$, so $\mathcal{Q}$ is complete. This completes the proof.

The following definition provides a measure of the "(cosine of the) angle between two subspaces".

DEFINITION. For noncomparable subspaces $M, N$ of $H$ define

$$
\alpha(M, N)=\sup \{|(x \mid y)|:\|x\|=\|y\|=1, x \in M \ominus M \cap N, y \in N \ominus M \cap N\} .
$$

Clearly $0 \leq \alpha(M, N) \leq 1$ and $\alpha(M, N)=0$ if and only if $M \ominus M \cap N$ and $N \ominus M \cap N$ are orthogonal. It is easy to show that $\alpha(M, N)<1$ if and only if the vector sum $M+N$ is closed. Note that $\|x+y\|^{2} \geq(1-\alpha(M, N))\left(\|x\|^{2}+\|y\|^{2}\right)$ for every $x \in M \ominus M \cap N$ and every $y \in N \ominus M \cap N$.

LEMMA 2. Let $H$ be infinite dimensional and let $\phi: C(H) \rightarrow C(H)$ be an automorphism. There exists $a \geq 1$ such that, for every pair of subspaces $M, N \in C(H)$ satisfying $M \neq(0), N \neq(0), M \cap N=(0)$,

$$
\alpha(\phi(M), \phi(N)) \leq\left(\frac{a-1+\alpha(M, N)}{a+1-\alpha(M, N)}\right)^{1 / 2}
$$

and

$$
\alpha(M, N) \leq\left(\frac{a-1+\alpha(\phi(M), \phi(N))}{a+1-\alpha(\phi(M), \phi(N))}\right)^{1 / 2}
$$

PROOF. By a result of [9], there exists a bicontinuous, linear or conjugate linear, bijection $S: H \rightarrow H$ such that $\phi(K)=S K$ for every $K \in C(H)$. Let $a=\|S\|^{2}\left\|S^{-1}\right\|^{2}$. Let $M, N \in C(H)$ be nonzero and satisfy $M \cap N=(0)$. Let $x \in M$ and $y \in N$ satisfy $\|S x\|=\|S y\|=1$ and put $\gamma=(S x \mid S y)$. Then $\|S x-\gamma S y\|^{2}=1-|\gamma|^{2}$. If $S$ is linear, then

$$
\|x-\gamma y\|^{2}=\left\|S^{-1}(S x-\gamma S y)\right\|^{2} \leq\left\|S^{-1}\right\|^{2}\|S x-\gamma S y\|^{2},
$$

so

$$
1-|\gamma|^{2} \geq\left(1 /\left\|S^{-1}\right\|^{2}\right)\|x-\gamma y\|^{2} .
$$

If $S$ is conjugate linear,

$$
\|x-\bar{\gamma} y\|^{2}=\left\|S^{-1}(S x-\gamma S y)\right\|^{2} \leq\left\|S^{-1}\right\|^{2}\|S x-\gamma S y\|^{2},
$$

so

$$
1-|\gamma|^{2} \geq\left(1 /\left\|S^{-1}\right\|^{2}\right)\|x-\bar{\gamma} y\|^{2} .
$$

Since $\|u+v\|^{2} \geq(1-\alpha(M, N))\left(\|u\|^{2}+\|v\|^{2}\right)$ for every $u \in M, v \in N$ we obtain

$$
1-|\gamma|^{2} \geq \frac{1}{\left\|S^{-1}\right\|^{2}}(1-\alpha(M, N))\left(\|x\|^{2}+|\gamma|^{2}\|y\|^{2}\right)
$$


whether $S$ is linear or conjugate linear. Since $1=\|S x\| \leq\|S\|\|x\|$ and $1=\|S y\| \leq$ $\|S\|\|y\|$, it follows that

$$
1-|\gamma|^{2} \geq(1 / a)(1-\alpha(M, N))\left(1+|\gamma|^{2}\right)
$$

From this,

$$
|(S x \mid S y)|^{2}=|\gamma|^{2} \leq \frac{a-1+\alpha(M, N)}{a+1-\alpha(M, N)}
$$

Thus,

$$
\alpha(\phi(M), \phi(N)) \leq\left(\frac{a-1+\alpha(M, N)}{a+1-\alpha(M, N)}\right)^{1 / 2},
$$

and the other inequality follows by applying this to $\phi^{-1}$.

Notice that every automorphism $\phi: C(H) \rightarrow C(H)$ preserves closed vector sums in the sense that, if $M, N \in C(H)$ and $M+N$ is closed, then $\phi(M)+\phi(N)$ is also closed. Indeed, if $\operatorname{dim} H<\infty$ the result is obvious. If $H$ is infinite dimensional, the result can be obtained as a consequence of the preceding lemma using the fact that, for incomparable subspaces $K$ and $L, K+L$ is closed if and only if $\alpha(K, L)<1$. Alternatively, the result in this case follows almost directly from the fact that $\phi$ is spatially induced by a bicontinuous, linear or conjugate linear, bijection $S$ :

$$
\phi(M)+\phi(N)=S M+S N=S(M+N)=S(M \vee N)=\phi(M \vee N)
$$

THEOREM 3. Let $B$ be a Boolean algebra subspace lattice on $H$. The following four conditions are equivalent.

(1) $B=S \mathcal{L}$ for some invertible operator $S \in B(H)$ and some commutative subspace lattice $\mathcal{L}$ on $H$.

(2) B satisfies $(\mathrm{AC})$.

(3) There exists $b \in[0,1)$ such that $\alpha(M, N) \leq b$ whenever $M, N \in B$ and $M \neq(0), N \neq(0), M \cap N=(0)$.

(4) For every $K, L \in B, K+L$ is closed.

Proof. Obviously (1) implies (2) since the map $M \rightarrow S M(M \in C(H))$ is an automorphism.

Suppose (2) is true. If $\operatorname{dim} H<\infty$, then $B$ is finite since every distributive lattice of finite length is finite [7, Theorem 148]. Since $\alpha(M, N)<1$ for every pair of incomparable elements $M, N \in B$, (3) is true. Now suppose $H$ is infinite dimensional and let $B=\phi(\mathcal{L})$ with $\phi$ an automorphism and $\mathcal{L}$ a commutative subspace lattice. Let $M, N \in B$ satisfy $M \neq(0), N \neq(0)$ and $M \cap N=(0)$. Let $M_{0}=\phi^{-1}(M)$ and $N_{0}=\phi^{-1}(N)$. Then $M_{0}, N_{0} \in \mathcal{L}$ and $M_{0} \neq(0), N_{0} \neq(0)$. Since the orthogonal projections with ranges $M_{0}$ and $N_{0}$ commute and $M_{0} \cap N_{0}=$ $(0), M_{0}$ is orthogonal to $N_{0}$. Thus $\alpha\left(M_{0}, N_{0}\right)=0$ and by Lemma $2, \alpha(M, N) \leq$ $((a-1) /(a+1))^{1 / 2}$, where $a \geq 1$ depends only on $\phi$. Taking $b=((a-1) /(a+1))^{1 / 2}$, (3) is true.

Suppose (3) is true. Let $K, L \in B$. If $K$ and $L$ are comparable, then $K+L$ is closed. Suppose $K, L$ are noncomparable. Then $K \neq(0), L \cap K^{\prime} \neq(0)$ (since $\left.L=\left(L \cap K^{\prime}\right) \vee(L \cap K)\right)$ and $K \cap\left(L \cap K^{\prime}\right)=(0)$, so $\alpha\left(K, L \cap K^{\prime}\right) \leq b<1$. Thus $K+\left(L \cap K^{\prime}\right)$ is closed. Now

$$
K+\left(L \cap K^{\prime}\right)=K \vee\left(L \cap K^{\prime}\right)=(K \vee L) \cap\left(K \vee K^{\prime}\right)=K \vee L,
$$


so $K \vee L \subseteq K+L$. Hence $K+L=K \vee L$, and (4) is true.

Finally, suppose (4) is true. In particular, $K+K^{\prime}$ is closed for every $K \in B$. For each $K \in B$ let $Q_{K}$ denote the (not necessarily orthogonal) projection onto $K$ along $K^{\prime}$. By Proposition $1, \mathcal{Q}=\left\{Q_{K}: K \in B\right\}$ is a complete Boolean algebra of projections in $H$. By Theorem 2.2 of [3] (see also [8, p. 2196]), $\mathcal{Q}$ is bounded. By Lemma 2 of $[8$, p. 1947], there exists an invertible operator $S \in B(H)$ such that $S^{-1} Q_{K} S$ is an orthogonal projection operator for every $K \in B$. Clearly, $S^{-1} Q_{K} S=P_{S^{-1} K}$ and $\mathcal{L}=S^{-1} B$ is a commutative subspace lattice. Since $B=S \mathcal{L},(1)$ is true. This completes the proof. The following result is a consequence of Lemma 6 of $[8$, p. 2199].

LEMMA 4 (W. G. BADE [2]). A complete Boolean algebra of projections in $H$ contains every idempotent operator in the weakly closed algebra it generates.

As a consequence we have the following result, in which $\mathfrak{A}^{\prime}$ denotes the commutant of $\mathfrak{A}$.

Proposition 5. If $\mathfrak{A} \subseteq B(H)$ is an abelian von Neumann algebra, then $B=$ Lat $\mathfrak{A}^{\prime}$ is a commutative Boolean algebra subspace lattice on $H$ and every commutative Boolean algebra subspace lattice on $H$ arises in this way.

Proof. First, let $B=$ Lat $\mathfrak{A}^{\prime}$ with $\mathfrak{A} \subseteq B(H)$ an abelian von Neumann algebra. It is easy to show that $B$ is a subspace lattice. Since $M \in B$ if and only if $P_{M} \in \mathfrak{A}$, $B$ is commutative and so distributive. Also $M \in B$ implies $M^{\perp} \in B$, so $B$ is complemented.

Conversely, let $B$ be a commutative Boolean algebra subspace lattice on $H$. We show that $B=$ Lat $\mathfrak{A}^{\prime}$, where $\mathfrak{A}=\left\{P_{K}: K \in B\right\}^{\prime \prime}$ is the von Neumann algebra generated by $\left\{P_{K}: K \in B\right\}$. Now $\mathfrak{A}^{\prime}=\left\{P_{K}: K \in B\right\}^{\prime}$, and it is clear that $B \subseteq$ Lat $\mathfrak{A}^{\prime}$. Let $M \in$ Lat $\mathfrak{A}^{\prime}$. Then $P_{M} \in \mathfrak{A}$. For every $K, L \in \mathcal{B}, P_{K}+P_{L}-P_{K} P_{L}$ is an orthogonal projection with range $K+L$, so $K+L$ is closed. Also, $K^{\prime}=K^{\perp}$ for every $K \in B$. By Proposition $1,\left\{P_{K}: K \in B\right\}$ is a complete Boolean algebra of projections in $H$. Since $\mathfrak{A}$ is the weakly closed algebra generated by $\left\{P_{K}: K \in B\right\}$, $P_{M} \in\left\{P_{K}: K \in B\right\}$ by Lemma 4 . Hence $M \in B$ and $B=$ Lat $\mathfrak{A}^{\prime}$. This completes the proof.

COROLlaRY 5.1. Every commutative Boolean algebra subspace lattice on $H$ is reflexive.

ProOF. For any subset $A \subseteq B(H)$, Lat $A$ is reflexive [10].

COROLlaRY 5.2. Every commutative subspace lattice on $H$ is included in some commutative Boolean algebra subspace lattice.

ProOF. Let $\mathcal{L}$ be a commutative subspace lattice on $H$. Let $\mathfrak{A}=\left\{P_{K}: K \in \mathcal{L}\right\}^{\prime \prime}$ be the von Neumann algebra generated by $\left\{P_{K}: K \in \mathcal{L}\right\}$. Then $\mathfrak{A}$ is abelian and clearly $\mathcal{L} \subseteq$ Lat $\mathfrak{A}^{\prime}$.

Since $H$ is separable, the preceding corollary can be proved by using Arveson's representation theorem for commutative subspace lattices [1, Theorem 1.3.1]. By this theorem, if $\mathcal{L}$ is a strongly closed commutative subspace lattice on $H$, there exist a compact metric space $X$, a finite positive Borel measure $m$ on $X$ and a unitary mapping $U: H \rightarrow L^{2}(X, m)$ such that $U \mathcal{L} \subseteq B$, where $B$ is the strongly closed commutative Boolean algebra subspace lattice on $L^{2}(X, m)$ consisting of the 
ranges of those orthogonal projections $P_{E}: L^{2} \rightarrow L^{2}$, where $E$ is a Borel subset of $X$ and $P_{E}$ is multiplication by the characteristic function $\chi_{E}$ of $E$. In [10] it is shown that, whether $H$ is separable or not, every strongly closed lattice of subspaces of $H$ is complete. We now use the above-mentioned representation theorem to point out that the converse is true, on a separable space, for commutative lattices.

PROPOSITION 6. Let $X$ be a compact metric space and let $m$ be a finite, positive Borel measure on $X$. Let $B$ be the strongly closed Boolean algebra subspace lattice on $L^{2}(X, m)$ consisting of the ranges of multiplications by characteristic functions of Borel subsets of $X$. Every complete lattice $₹$ of subspaces of $L^{2}(X, m)$ satisfying $\mathcal{F} \subseteq B$ is strongly closed.

Proof. Since $L^{2}(X, m)$ is separable, it is enough to show that if $\left(P_{n}\right)_{1}^{\infty}$ is a sequence of orthogonal projections, with $R\left(P_{n}\right) \in \mathcal{F}$ for every $n \geq 1$, which converges strongly to an operator $T$, then $T$ is an orthogonal projection and $R(T) \in$ F. Clearly $T$ must be an orthogonal projection, and since $B$ is strongly closed, $R(T) \in B$. Let $P_{n}$ be multiplication by the characteristic function of the Borel set $E_{n}$, and let $T$ be multiplication by the characteristic function of the Borel set $E$.

Since $m$ is finite, the constant function 1 belongs to $L^{2}(X, m)$ and since $P_{n} \rightarrow T$ strongly,

$$
\left\|\left(P_{n}-T\right) 1\right\|^{2}=\int\left|\chi_{E_{n}}-\chi_{E}\right|^{2} d m=m\left(E_{n} \triangle E\right) \rightarrow 0 \quad \text { as } n \rightarrow \infty .
$$

By passing to a subsequence if necessary, we may assume that $m\left(E_{n} \Delta E\right) \leq 2^{-n}$, $n \geq 1$. Put $E_{\infty}=\bigcap_{n=1}^{\infty} \bigcup_{k=n}^{\infty} E_{k}$ and let $P_{E_{\infty}}$ denote multiplication by the characteristic function of $E_{\infty}$. Since $\mathcal{F}$ is complete, $R\left(P_{E_{\infty}}\right) \in \mathcal{F}$. But $m\left(E_{\infty} \triangle E\right)=0$, so $P_{E_{\infty}}=T$. Thus $R(T) \in \mathcal{F}$, and the proof is complete.

COROLLARY 6.1. Every commutative complete lattice of subspaces of a complex separable Hilbert space $H$ is \$trongly closed. In particular, every commutative subspace lattice on $H$ is strongly closed.

ProOF. Let $\mathcal{L}$ be a commutative complete lattice of subspaces of $H$. We can assume that $(0), H \in \mathcal{L}$. If $\overline{\mathcal{L}}$ denotes the set of subspaces $M$ of $H$ for which $P_{M}$ belongs to the strong closure of $\left\{P_{N}: N \in \mathcal{L}\right\}$, then $\overline{\mathcal{L}}$ is a commutative strongly closed subspace lattice on $H$. By Arveson's representation theorem, there exist $X, m$ and $B$ as in the statement of the proposition and a unitary mapping $U: H \rightarrow L^{2}(X, m)$ such that $U \overline{\mathcal{L}} \subseteq B$. By the proposition, $U \mathcal{L}$ is strongly closed, so $U \mathcal{L}=U \overline{\mathcal{L}}$. Thus $\mathcal{L}=\overline{\mathcal{L}}$, and the proof is complete.

By Theorem 1.6 .3 of $[\mathbf{1}]$, it now follows that every commutative subspace lattice on $H$ is reflexive. Corollary 5.1 is a special instance of this.

PROPOSITION 7. Let $₹$ be a subspace lattice on $H$. Then $₹$ satisfies (AC) if and only if $₹ \subseteq B$ for some Boolean algebra subspace lattice on $H$ satisfying (AC).

ProOF. Suppose first that $\mathcal{F}=\phi(\mathcal{L})$ for some automorphism $\phi$ and some commutative subspace lattice $\mathcal{L}$. By Corollary $5.2, \mathcal{L} \subseteq B_{0}$ for some commutative Boolean algebra subspace lattice $B_{0}$. Thus $₹ \subseteq B=\phi\left(B_{0}\right)$.

Conversely, suppose $₹ \subseteq B$ where $B=\phi\left(B_{0}\right)$ is a Boolean algebra subspace lattice, $\phi$ is an automorphism and $B_{0}$ is commutative. Then $\mathcal{F}=\phi(\mathcal{L})$, where $\mathcal{L}=\phi^{-1}(\mathcal{F}) \subseteq B_{0}$ is a commutative subspace lattice. 
COROLlaRY 7.1. Let $\mp$ be a subspace lattice on $H$. The following conditions are equivalent.

(1) $\mathcal{I}=S \mathcal{L}$ for some positive invertible operator $S \in B(H)$ and some commutative subspace lattice $\mathcal{L}$ on $H$.

(2) $\mp$ satisfies (AC).

(3) $\exists \subseteq B$ for some Boolean algebra subspace lattice $B$ on $H$ with the property that $K+L$ is closed for every $K, L \in B$.

Proof. Obviously (1) implies (2). Also, (2) and (3) are equivalent by Theorem 3 and Proposition 7 . We now show that (2) implies (1). Suppose (2) is true. By

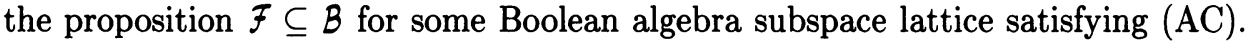
By Theorem 3, there exists an invertible operator $T \in B(H)$ such that $T^{-1} B$ is commutative. Let $T^{*}=U S$ be the polar decomposition of $T^{*}$. Then $U$ is unitary and $S$ is positive and invertible. Now $\mathcal{F}=S \mathcal{L}$, where $\mathcal{L}=S^{-1} \mathcal{F}=U^{*}\left(T^{-1} \mathcal{F}\right) \subseteq$ $U^{*}\left(T^{-1} B\right)$ is commutative. The proof is complete.

The characterization of (AC) provided by the equivalence of conditions (2) and (3) of the preceding corollary is somewhat unsatisfactory because condition (3) is not intrinsic to $\mathcal{F}$. If $\mathcal{F}$ is a Boolean algebra, Theorem 3 provides an intrinsic necessary and sufficient condition for (AC). Intrinsic characterisations of $(\mathrm{AC})$ can also be found if $\mathcal{F}$ is finite or if $H$ is finite dimensional, as we now show.

THEOREM 8. A finite subspace lattice $\mathcal{F}$ on $H$ satisfies (AC) if and only if $\mathcal{F}$ is distributive and $K+L$ is closed for every $K, L \in \mathcal{F}$.

ProOF. Let $₹$ be a finite subspace lattice on $H$. If $\mp$ satisfies (AC), then $₹$ is distributive and $K+L$ is closed for every $K, L \in \mathcal{F}$, by Corollary 7.1.

Conversely, suppose $\mathcal{F}$ is distributive and $K+L$ is closed for every $K, L \in \mathcal{F}$. We show that $₹ \subseteq B$ for some Boolean algebra subspace lattice $B$ on $H$ with the property that $K+L$ is closed for every $K, L \in B$. Then $₹$ satisfies (AC) by Corollary 7.1. Let $K_{1}, K_{2}, \ldots, K_{n}$ be the nonzero join-irreducible elements of ₹. For $j=1,2, \ldots, n$ let $K_{j}^{0}$ be the unique element of $₹$ covered by $K_{j}$ and put $H_{j}=K_{j} \ominus K_{j}^{0}$. For each nonzero element $M \in \mathcal{F}$ set $\Omega(M)=\left\{j: K_{j} \subseteq M\right\}$. Then [7, Theorem 147] the cardinality of $\Omega(M)$ is the height of $M$ in $₹$ and $M=$ $\bigvee\left\{K_{j}: j \in \Omega(M)\right\}$. Call a finite set of subspaces $\left\{N_{1}, N_{2}, \ldots, N_{s}\right\}$ of $H$ a basis for the subspace $N$ of $H$ if $N_{j} \subseteq N, j=1,2, \ldots, s$, and every vector $x \in N$ has a unique representation $x=\sum_{j=1}^{s} x_{j}$ with $x_{j} \in N_{j}, j=1,2, \ldots, s$. We show that, for every nonzero subspace $M \in \mathcal{F},\left\{H_{j}: j \in \Omega(M)\right\}$ is a basis for $M$. The proof is by induction on the cardinality of $\Omega(M)$.

Suppose $\operatorname{card} \Omega(M)=1$. Then $M=K_{m}$ for some $1 \leq m \leq n$ and $K_{m}^{0}=(0)$. Thus $H_{m}=K_{m}=M$ and $\left\{H_{m}\right\}$ is clearly a basis for $M$.

Now suppose $\left\{H_{j}: j \in \Omega(N)\right\}$ is a basis for $N$ whenever card $\Omega(N) \leq k$, and suppose $\operatorname{card} \Omega(M)=k+1$. Now $M=\bigvee\left\{K_{j}: j \in \Omega(M)\right\}$, so $H_{j} \subseteq M$ for every $j \in \Omega(M)$. Let $N$ be any element of $₹$ covered by $M$. Since the height of $N$ is one less than that of $M, \operatorname{card} \Omega(N)=k$ and $\Omega(M)=\Omega(N) \cup\{t\}$ for some $t \in\{1,2, \ldots, n\}$ for which $K_{t} \nsubseteq N$. Since $K_{t}+N=M$ covers $N$, it follows that [7, Theorem 91] $K_{t}$ covers $K_{t} \cap N$. Thus $K_{t} \cap N=K_{t}^{0}$. Let $x \in M$. There are unique vectors $y \in H_{t}$ and $z \in N$ such that $x=y+z$. By the induction hypothesis $z$ has the unique representation $z=\sum\left\{z_{j}: j \in \Omega(N)\right\}$ with $z_{j} \in H_{j}, j \in \Omega(N)$. It follows that $x=y+\sum\left\{z_{j}: j \in \Omega(N)\right\}$ is the required unique representation of 


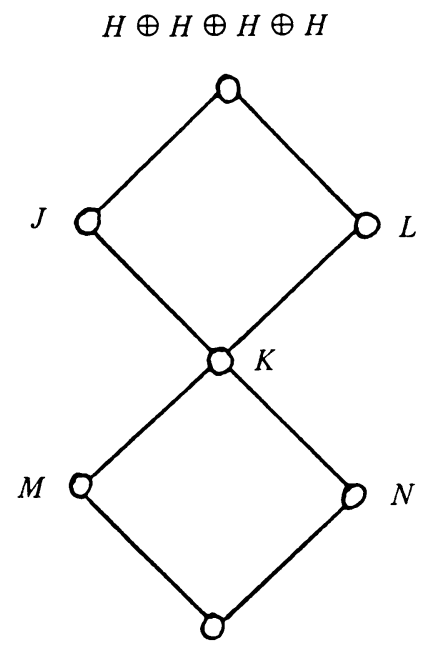

(0)

FIGURE 1

$x$. Thus $\left\{H_{j}: j \in \Omega(M)\right\}$ is a basis for $M$, and by the principle of induction this is true for every nonzero element $M$ of $\mathcal{F}$.

In particular, since $\Omega(H)=\{1,2, \ldots, n\}$, each vector $x \in H$ has a unique representation $x=\sum_{j=1}^{n} x_{j}$ with $x_{j} \in H_{j}, j=1,2, \ldots, n$. Consider the map $T$ from the direct sum $H_{1} \oplus H_{2} \oplus \cdots \oplus H_{n}$ into $H$ defined by $T\left(x_{1}, x_{2}, \ldots, x_{n}\right)=\sum_{j=1}^{n} x_{j}$. This $T$ is linear, bijective and bounded, so by the Open Mapping Theorem, $T^{-1}$ is bounded. Thus there is a constant $c>0$ satisfying

$$
\sum_{j=1}^{n}\left\|x_{j}\right\|^{2} \leq c\left\|\sum_{j=1}^{n} x_{j}\right\|^{2}, \quad \text { for any } x_{j} \in H_{j}, j=1,2, \ldots, n \text {. }
$$

For $j=1,2, \ldots, n$ define $Q_{j}: H \rightarrow H$ by $Q_{j} x=Q_{j}\left(\sum_{k=1}^{n} x_{k}\right)=x_{j}$. Then each $Q_{j}$ is linear, idempotent and because of $(*)$, bounded. Also $Q_{j} Q_{k}=0$ if $j \neq k$, and $\sum_{j=1}^{n} Q_{j}=1$. For each subset $\mathcal{E} \subseteq\{1,2, \ldots, n\}$ define $Q_{\mathcal{E}}=\sum\left\{Q_{j}: j \in \mathcal{E}\right\}$, with $Q_{\phi}=0$ and define $M_{\mathcal{E}}=R\left(Q_{\mathcal{E}}\right)$. The set $\mathcal{Q}=\left\{Q_{\mathcal{E}}: \mathcal{E} \subseteq\{1,2, \ldots, n\}\right\}$ is a complete (finite) Boolean algebra of projections, so by Proposition $1, B=\left\{M_{\mathcal{E}}: \mathcal{E} \subseteq\right.$ $\{1,2, \ldots, n\}\}$ is a (finite) Boolean algebra subspace lattice on $H$ with the property that $K+L$ is closed for every $K, L \in B$.

Finally we note that $\mathcal{F} \subseteq B$, since for every nonzero subspace $M \in \mathcal{F}$ we have $M=M_{\Omega(M)}$. This completes the proof.

COROllaRY 8.1. Let $H$ be finite dimensional. A subspace lattice $₹$ on $H$ satisfies $(A C)$ if and only if it is distributive.

PROOF. All vector sums are closed in finite dimensions. If $₹$ satisfies (AC) it is distributive by Corollary 7.1. Conversely, if $\mathcal{F}$ is distributive then $\mathcal{F}$ is finite [7, Theorem 148]. Hence $₹$ satisfies (AC) by the above theorem.

Note that, unlike the case for a Boolean algebra, for a finite distributive subspace lattice $₹$ on $H$ the condition "there exists $b \in[0,1)$ such that $\alpha(M, N) \leq b$ whenever 
$M, N \in \mathcal{F}$ and $M \neq(0), N \neq(0), M \cap N=(0)$ " is not equivalent to $₹$ satisfying (AC). This condition is necessary for (AC), since $\mathcal{F}$ has closed vector sums if it satisfies (AC), but not sufficient, as the following simple example shows.

EXAMPLE. Let $H$ be infinite dimensional. Let $A \in B(H)$ be an injective operator with dense but nonclosed range. Define the subspaces $J, K, L, M, N$ of $H \oplus H \oplus H \oplus H$ by $J=H \oplus H \oplus H \oplus(0), K=H \oplus H \oplus(0) \oplus(0), L=$ $\{(x, y, z, A z): x, y, z \in H\}, M=H \oplus(0) \oplus(0) \oplus(0), N=(0) \oplus H \oplus(0) \oplus(0)$. Then $\mathcal{F}=\{(0), J, K, L, M, N, H \oplus H \oplus H \oplus H\}$ is a finite distributive subspace lattice on $H \oplus H \oplus H \oplus H$. Figure 1 is a labelled Hasse diagram of $₹$.

Since $M$ and $N$ are orthogonal, $\alpha(M, N)=0$. However, $J+L$ is not closed, since if $x \notin R(A)$ then $(0,0,0, x) \notin J+L$. Thus $₹$ cannot satisfy (AC).

As noted earlier, every commutative subspace lattice on $H$ is reflexive. It follows that every subspace lattice $₹$ on $H$ satisfying $(\mathrm{AC})$ is reflexive. For, by Corollary 7.1 , if $\mathcal{F}$ satisfies $(\mathrm{AC})$, then $\mathcal{F}=S \mathcal{L}$ for some (positive) invertible operator $S \in$ $B(H)$ and some commutative subspace lattice $\mathcal{L}$. Thus $\operatorname{Alg} \mathcal{F}=S(\operatorname{Alg} \mathcal{L}) S^{-1}$ and Lat $\operatorname{Alg} \mathcal{F}=S(\operatorname{Lat} \operatorname{Alg} \mathcal{L})=S \mathcal{L}=\mathcal{F}$. It is shown in [5] that not every Boolean algebra subspace lattice on a Hilbert space is reflexive, and the author asks if every strongly closed Boolean algebra subspace lattice is reflexive. A partial answer is furnished by Theorem 3 and the fact that every reflexive subspace lattice on a Hilbert space is strongly closed [10]: Every Boolean algebra subspace lattice $B$ on $H$ with the property that $K+L$ is closed for every $K, L \in B$ is reflexive and strongly closed. In [9] it is shown that if $H$ is infinite dimensional (not necessarily separable) every automorphism $\phi$ of $C(H)$ preserves the property of being strongly

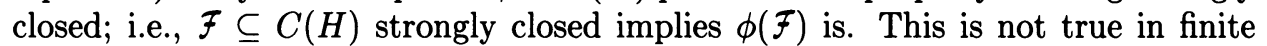
dimensions.

EXAMPLE. Let $2 \leq \operatorname{dim} H<\infty$. For any vector $z \in H$ let $\langle z\rangle$ denote the linear span of $\{z\}$. We will use the fact that if $z, z_{1}, z_{2}, z_{3}, \ldots$ are nonzero vectors of $H$, then $P_{\left\langle z_{n}\right\rangle} \rightarrow P_{\langle z\rangle}$ strongly if and only if there exists a sequence $\left(\mu_{n}\right)_{1}^{\infty}$ of scalars such that $\mu_{n} z_{n} /\left\|z_{n}\right\| \rightarrow z /\|z\|$. Let $\left\{e_{1}, e_{2}, \ldots, e_{m}\right\}$ be a basis of $H$. Let $\tau$ be a discontinuous field automorphism of C. Define $S: H \rightarrow H$ by $S x=S\left(\sum_{j=1}^{m} \alpha_{j} e_{j}\right)=$ $\sum_{j=1}^{m} \tau\left(\alpha_{j}\right) e_{j}$. Then $S$ is an additive bijection and $S(\alpha x)=\tau(\alpha) S x$ for every $x \in H$ and every scalar $\alpha$. The map $\phi: C(H) \rightarrow C(H)$ defined by $\phi(M)=S M$ is an automorphism of $C(H)$. Let $f \in H$ be a nonzero vector and let $\left(\lambda_{n}\right)_{1}^{\infty}$ be a sequence of nonzero complex numbers satisfying $\lambda_{n} \rightarrow 0, \tau\left(\lambda_{n}\right) \nrightarrow 0$. Now $\phi\langle f\rangle \neq H$, so there exists a unit vector $S e$ such that $(S f \mid S e)=0$. Now $e+\lambda_{n} f \rightarrow e$ and $e+\lambda_{n} f(n \geq 1)$ and $e$ are nonzero. Thus $P_{\left\langle e+\lambda_{n} f\right\rangle} \rightarrow P_{\langle e\rangle}$ strongly. Suppose that $P_{\phi\left\langle e+\lambda_{n} f\right\rangle} \rightarrow P_{\phi\langle e\rangle}$ strongly. Then there exists a sequence $\left(\mu_{n}\right)_{1}^{\infty}$ of scalars such that $\mu_{n} S\left(e+\lambda_{n} f\right) /\left\|S\left(e+\lambda_{n} f\right)\right\| \rightarrow S e$. Taking inner products with respect to $S e$ gives $\mu_{n} /\left\|S\left(e+\lambda_{n} f\right)\right\| \rightarrow 1$. Thus $S\left(e+\lambda_{n} f\right)=S e+\tau\left(\lambda_{n}\right) S f \rightarrow S e$, so $\tau\left(\lambda_{n}\right) \rightarrow 0$. This is a contradiction, so $\left(P_{\phi\left\langle e+\lambda_{n} f\right\rangle}\right)_{1}^{\infty}$ does not converge strongly to $P_{\phi\langle e\rangle}$. Hence the automorphism $\phi$ of $C(H)$ is not continuous in the induced strong operator topology (for every subspace $M$, identity $M$ with the orthogonal projection $P_{M}$ ), and so its inverse $\phi^{-1}$ is an automorphism which does not preserve the property of being strongly closed.

It is also shown in $[\mathbf{9}]$ that if $H$ is infinite dimensional (not necessarily separable) every automorphism $\phi$ of $C(H)$ preserves reflexivity and transitivity. It may be of some interest to note that this is true in finite dimensions as well. 
PROPOSITION 9. Let $H$ be finite dimensional and let $\phi$ be an automorphism of $C(H)$. For every nonempty subset $₹ \subseteq C(H)$, Lat $\operatorname{Alg} \phi(\mathcal{F})=\phi(\operatorname{Lat} A \lg \mathcal{F})$. Consequently $\mathcal{F}$ is reflexive (respectively, transitive) if and only if $\phi(\mathcal{F})$ is.

ProOF. First suppose that $3 \leq \operatorname{dim} H<\infty$. By the First Fundamental Theorem of Projective Geometry [4, p. 44], there exists a bijection $S: H \rightarrow H$ and a field automorphism $\tau$ of $\mathbb{C}$ satisfying $S(x+y)=S x+S y$ for every $x, y \in H$ and $S(\lambda x)=$ $\tau(\lambda) S x$ for every $x \in H$ and $\lambda \in \mathbb{C}$, with the property that $\phi(M)=S M$ for every $M \in C(H)$. The map $S^{-1}: H \rightarrow H$ is additive and $S^{-1}(\lambda x)=\tau^{-1}(\lambda) S^{-1} x$ for every $x \in H$ and every $\lambda \in \mathbb{C}$. It is not difficult to verify that $\operatorname{Alg} \phi(\mathcal{F})=S(\operatorname{Alg} \mathcal{F}) S^{-1}$ and that $\operatorname{Lat} A \lg \phi(\mathcal{F})=S(\operatorname{Lat} A \lg \mathcal{F})=\phi(\operatorname{Lat} A \lg \mathcal{F})$ for every nonempty subset $\xi \subseteq C(H)$.

If $\operatorname{dim} H=1$, Lat $\operatorname{Alg} \phi(\xi)=\phi(\operatorname{Lat} \operatorname{Alg} \varsubsetneqq)=\{(0), H\}$. Finally, suppose $\operatorname{dim} H$ $=2$. For any nonempty subset $\mathcal{G} \subseteq C(H)$, Lat $A \lg \mathcal{G}$ is

(i) $\{(0), H\}$ if $\mathcal{G}$ contains no element different from $(0), H$,

(ii) $\{(0), M, H\}$ if $\mathcal{G}$ contains precisely one element $M \neq(0), H$,

(iii) $\{(0), M, N, H\}$ if $\mathcal{G}$ contains precisely two elements $M, N \neq(0), H$,

(iv) $C(H)$ if $\mathcal{G}$ contains more than two elements different from $(0), H$.

From this, Lat $\operatorname{Alg} \phi(\xi)=\phi(\operatorname{Lat} A \lg \mp)$ for every nonempty subset $₹ \subseteq C(H)$.

3. The equation $T x=y$. Let $₹$ be a subspace lattice on $H$ and let $x, y \in H$. The condition

there exists $c \geq 0$ such that, for every $K \in \mathcal{F}$,

$$
\left\|\left(1-P_{K}\right) y\right\| \leq c\left\|\left(1-P_{K}\right) x\right\|
$$

is necessary for the existence of an operator $T \in \operatorname{Alg} \mp$ satisfying $T x=y$. For, if such a $T$ exists and $K \in \mathcal{F}$, then

$$
\left\|\left(1-P_{K}\right) y\right\|=\left\|\left(1-P_{K}\right) T x\right\|=\left\|\left(1-P_{K}\right) T\left(1-P_{K}\right) x\right\| \leq\|T\|\left\|\left(1-P_{K}\right) x\right\| .
$$

Condition $(\dagger)$ is not sufficient for $T x=y$ to have a solution $T \in \operatorname{Alg} \mathcal{F}$, even when $\mp$ is a very simple finite Boolean algebra.

EXAMPLE. Let $H$ be infinite dimensional. Let $A \in B(H)$ be an injective operator with dense but nonclosed range. Let $₹$ be the finite Boolean algebra subspace lattice on $H \oplus H$ with elements $(0), H \oplus H, G(0)=\{(\xi, 0): \xi \in H\}$ and $G(A)=\{(\xi, A \xi): \xi \in H\}$. Let $x=(0, A \eta)$ with $\eta \neq 0$ and let $y=(0, \varsigma)$ with $\varsigma$ not in the range of $A$. Condition $(\dagger)$ is satisfied since neither of $\left\|\left(1-P_{G(0)}\right) x\right\|$, $\left\|\left(1-P_{G(A)}\right) x\right\|,\|x\|$ is zero. However, there does not exist $T \in A \operatorname{Alg} \mp$ such that $T x=y$. For let $T \in \operatorname{Alg} \mathcal{F}$. Then $T=\left[\begin{array}{cc}X & Z \\ 0 & Y\end{array}\right]$, with $X, Y, Z \in B(H)$ satisfying $Y A=A(X+Z A)$. Thus $T x=(Z A \eta, A(X+Z A) \eta)$. Since $A(X+Z A) \eta$ belongs to the range of $A$, we cannot have $T x=y$.

Recently, it has been shown $[\mathbf{1 1}]$ that condition $(\dagger)$ is also sufficient for the existence of $T \in \operatorname{Alg} \mp$ satisfying $T x=y$, provided that $₹$ is commutative.

PROPOSITION 10. Let $\mp$ be a finite distributive subspace lattice on $H$ with the property that $K+L$ is closed for every $K, L \in \mathcal{F}$. Let $x, y \in H$. There exists an operator $T \in \operatorname{Alg} \mp$ such that $T x=y$ if and only if condition $(\dagger)$ is satisfied.

PROOF. By Theorem 8 and Corollary $7.1, \mathcal{F}=S \mathcal{L}$ for some (positive) invertible operator $S \in B(H)$ and some commutative subspace lattice $\mathcal{L}$ on $H$. Now $\operatorname{Alg} \mathcal{F}=$ 
$S(\operatorname{Alg} \mathcal{L}) S^{-1}$, and clearly, $T x=y$ has a solution $T \in \operatorname{Alg} \mathcal{F}$ if and only if $A S^{-1} x=$ $S^{-1} y$ has a solution $A \in \operatorname{Alg} \mathcal{L}$. By the theorem of [11], there exists such an $A$ if and only if there exists $c \geq 0$ such that

$$
\left\|\left(1-P_{S^{-1} K}\right) S^{-1} y\right\| \leq c\left\|\left(1-P_{S^{-1} K}\right) S^{-1} x\right\| \quad \text { for every } K \in \mathcal{F} .
$$

Since $\mathcal{F}$ is finite, such a $c$ exists if and only if $K \in \mathcal{F}$ and $\left(1-P_{S^{-1} K}\right) S^{-1} x=0$ implies $\left(1-P_{S^{-1} K}\right) S^{-1} y=0$. The latter is equivalent to $y \in \bigcap\{K \in \mathcal{F}: x \in K\}$, which is, in turn, equivalent to condition $(\dagger)$. This completes the proof.

PROPOSITION 11. Let $B$ be a Boolean algebra subspace lattice on $H$ with the property that $K+L$ is closed for every $K, L \in B$. Let $x, y \in H$. There exists $T \in \operatorname{Alg} B$ satisfying $T x=y$ if and only if there exists $c \geq 0$ such that, for every $K \in B,\left\|\left(1-Q_{K}\right) y\right\| \leq c\left\|\left(1-Q_{K}\right) x\right\|$ (where $Q_{K}$ denotes the projection onto $K$ along its complement $\left.K^{\prime}\right)$.

Proof. Suppose first that $T x=y$ has a solution $T \in \operatorname{Alg} B$. Let $K \in B$. Then $T K \subseteq K$ and $T K^{\prime} \subseteq K^{\prime}$ gives $T Q_{K}=Q_{K} T$. Thus

$$
\left\|\left(1-Q_{K}\right) y\right\|=\left\|\left(1-Q_{K}\right) T x\right\|=\left\|T\left(1-Q_{K}\right) x\right\| \leq\|T\|\left\|\left(1-Q_{K}\right) x\right\|,
$$

so we may take $c=\|T\|$.

Conversely, suppose such a $c \geq 0$ exists. By Theorem $3, B=S \mathcal{L}$ for some invertible operator $S \in B(H)$ and some commutative subspace lattice $\mathcal{L}$ on $H$. For every $K \in B, S^{-1} Q_{K} S=P_{S^{-1} K}, S^{-1}\left(1-Q_{K}\right) S=1-P_{S^{-1} K}$ and

$$
\begin{aligned}
\left\|\left(1-P_{S^{-1} K}\right) S^{-1} y\right\| & =\left\|S^{-1}\left(1-Q_{K}\right) y\right\| \leq\left\|S^{-1}\right\|\left\|\left(1-Q_{K}\right) y\right\| \\
& \leq\left\|S^{-1}\right\| c\left\|\left(1-Q_{K}\right) x\right\| \leq\left\|S^{-1}\right\| c\|S\|\left\|S^{-1}\left(1-Q_{K}\right) x\right\| \\
& =\left(c\|S\|\left\|S^{-1}\right\|\right)\left\|\left(1-P_{S^{-1} K}\right) S^{-1} x\right\| .
\end{aligned}
$$

By the theorem of $[\mathbf{1 1}]$, there exists $A \in \operatorname{Alg} \mathcal{L}$ such that $A S^{-1} x=S^{-1} y$. Then $T=S A S^{-1} \in \operatorname{Alg} B$ and $T x=y$. This completes the proof.

ADDENDUM. After the completion of this manuscript it came to the authors' attention that two recent preprints contain some intersection with the results presented here. Theorem 4.3 of Perturbations of finite dimensional operator algebras by M. D. Choi and K. R. Davidson is essentially our Corollary 8.1. Also, Proposition 1.2 of Perturbations of reflexive operator algebras by K. R. Davidson is almost the same as our Proposition 7. The authors thank the referee for his comments.

\section{REFERENCES}

1. W. B. Arveson, Operator algebras and invariant subspaces, Ann. of Math. (2) 100 (1974), 433-532.

2. W. G. Bade, Weak and strong limits of spectral operators, Pacific J. Math. 4 (1954), $393-413$

3. __ On Boolean algebras of projections and algebras of operators, Trans. Amer. Math. Soc. 80 (1955), 345-360.

4. R. Baer, Linear algebra and projective geometry, Academic Press, New York, 1952.

5. J. B. Conway, A complete Boolean algebra of subspaces which is not reflexive, Bull. Amer. Math. Soc. 79 (1973), 720-722.

6. K. R. Davidson, Commutative subspace lattices, Indiana Univ. Math. J. (3) 27 (1978), 479-490.

7. T. Donnellan, Lattice theory, Pergamon Press, London, 1968. 
8. N. Dunford and J. T. Schwartz, Linear operators. Part III: Spectral operators, Wiley, New York, 1971.

9. P. A. Fillmore and W. E. Longstaff, On isomorphisms of lattices of closed subspaces, Canad. J. Math. (5) 36 (1984), 820-829.

10. P. R. Halmos, Reflexive lattices of subspaces, J. London Math. Soc. 4 (1971), 257-263.

11. A. Hopenwasser, The equation $T x=y$ in a reflexive operator algebra, Indiana Univ. Math. J. (1) 29 (1980), 121-126.

SChool of Mathematical and Physical Sciences, Murdoch University, MuRDoCh, Western Australia 6153, Australia

Department of Mathematics, University of Western AUstralia, Nedlands, Western Australia 6009, AUstralia 